\title{
Phase space view of quantum mechanical systems and Fisher information
}

\author{
Á. Nagy \\ Department of Theoretical Physics, University of Debrecen, H-4010 Debrecen, Hungary
}

(Dated: May 2, 2016)

\begin{abstract}
Pennini and Plastino showed that the form of the Fisher information generated by the canonical distribution function reflects the intrinsic structure of classical mechanics. Now, a quantum mechanical generalization of the Pennini - Plastino theory is presented based on the thermodynamical transcription of the density functional theory. Comparing to the classical case, the phase-space Fisher information contains an extra term due to the position dependence of the temperature. However, for the special case of constant temperature, the expression derived bears resemblance to the classical one. A complete analogy to the classical case is demonstrated for the linear harmonic oscillator.
\end{abstract}




\section{INTRODUCTION}

Fisher information [1] has received a growing interest in physics for about twenty years [2]. A decade ago Pennini and Plastino [3] showed that the form of the Fisher information generated by the canonical distribution function reflects the intrinsic structure of classical mechanics. They proved that the Fisher information has a universal form containing the derivatives of the generalized coordinates and momenta with respect to time.

A "thermodynamical" interpretation of ground-state quantum mechanics is possible on the grounds of density functional theory. About thirty years ago Ghosh, Berkowitz and Parr published a paper entitled "Transcription of ground-state density functional theory into a local thermodynamics" [4]. They found a phase-space distribution function $f(\mathbf{r}, \mathbf{p})$ by maximizing a phase-space Shannon information entropy subject to the conditions that $f$ yields the density $n(\mathbf{r})$ and the local kinetic energy density of the system. A local MaxwellBoltzmann distribution function was obtained and the concept of local temperature was developed. Several approximate expressions for important quantities such as exchange energy were derived [5]. Local entropy, free energy were defined and the analogy with the classical thermodynamics of fluids was founded [6]. The formalism has been generalized in several ways [7-17].

Here a quantum mechanical generalization of the Pennini - Plastino theory is presented utilizing the thermodynamical transcription of the density functional theory. The form of the phase-space Fisher information is not exactly the same as the classical one as there is an extra term due to the position dependence of the temperature. However, if the temperature is constant, a form resembling to the classical one is gained. A complete analogy to the classical case is demonstrated for the linear harmonic oscillator.

In the following section the Pennini - Plastino theory is summarised. Section III. gives the gist of the thermodynamical interpretation of ground-state quantum mechanics. Section IV. presents the new theory: the phase-space Fisher information based on density functional theory. The last section is devoted to illustrative examples and discussion. 


\section{CLASSICAL SYSTEMS}

First the classical results based on the work of Pennini and Plastino [3] are summarized. Consider a classical system with degrees of freedom $l$. The phase-space coordinates are denoted by $\mathbf{z}=\left(q_{1}, \ldots q_{l}, p_{1}, \ldots, p_{l}\right)$. In thermal equilibrium at the temperature $T$ the canonical probability density is

$$
\tilde{\varrho}(\mathbf{z})=\frac{e^{-\tilde{\beta} H(\mathbf{z})}}{Z}
$$

where $H$ is the Hamiltonian, $\tilde{\beta}=1 /(k T)$ and $k$ is Boltzmann's constant. The partition function reads

$$
Z=\int d \mathbf{z} e^{-\tilde{\beta} H(\mathbf{z})}
$$

The canonical equations

$$
\begin{gathered}
\dot{q}_{i}=\frac{\partial H(\mathbf{z})}{\partial p_{i}} \\
\dot{p}_{i}=-\frac{\partial H(\mathbf{z})}{\partial q_{i}}
\end{gathered}
$$

can be rewritten

$$
\begin{gathered}
\dot{q}_{i}=-k T \frac{\partial \ln \tilde{\varrho}(\mathbf{z})}{\partial p_{i}}, \\
\dot{p}_{i}=k T \frac{\partial \ln \tilde{\varrho}(\mathbf{z})}{\partial q_{i}}
\end{gathered}
$$

with $i=1, \ldots, l$. In deriving Eqs. (5) and (6) we utilized that

$$
\frac{\partial \ln \varrho(\mathbf{z})}{\partial p_{i}}=-\tilde{\beta} \frac{\partial H(\mathbf{z})}{\partial p_{i}}
$$

and

$$
\frac{\partial \ln \varrho(\mathbf{z})}{\partial q_{i}}=-\tilde{\beta} \frac{\partial H(\mathbf{z})}{\partial q_{i}}
$$

The phase space Fisher information has the form

$$
I=\sum_{i=1}^{l} \int d \mathbf{z} \tilde{\varrho}(\mathbf{z})\left(a\left[\frac{\partial \ln \tilde{\varrho}(\mathbf{z})}{\partial p_{i}}\right]^{2}+b\left[\frac{\partial \ln \tilde{\varrho}(\mathbf{z})}{\partial q_{i}}\right]^{2}\right),
$$


where the coefficients $a$ and $b$ are introduced for dimensional reasons [18]. Making use of Eqs. (7) and (8) we arrive at

$$
I=\tilde{\beta}^{2} \sum_{i=1}^{l} \int d \mathbf{z} \tilde{\varrho}(\mathbf{z})\left(a\left[\frac{\partial H(\mathbf{z})}{\partial p_{i}}\right]^{2}+b\left[\frac{\partial H(\mathbf{z})}{\partial q_{i}}\right]^{2}\right) .
$$

Substituting the canonical equations (3) and (4) into this form of the Fisher information the final expression is gained:

$$
I=\tilde{\beta}^{2} \sum_{i=1}^{l}\left(a\left\langle\dot{q}_{i}^{2}\right\rangle+b\left\langle\dot{p}_{i}^{2}\right\rangle\right) .
$$

There are several related papers in the subject, see e.g. [19-22].

\section{A "THERMODYNAMICAL" TRANSCRIPTION OF QUANTUM MECHAN- ICS}

To present the quantum mechanical generalization of the Pennini-Plastino theory, the Ghosh-Berkowitz-Parr transcription of the density functional theory [4] is utilized. In this section this "thermodynamical" transcription is summarized.

Consider a system of $N$ electrons moving in a local external potential $v(\mathbf{r})$. Based on the Hohenherg-Kohn theorems [23] $v(\mathbf{r})$ is determined within a trivial additive constant by the knowledge of the ground-state electron density $n(\mathbf{r})$ and the ground-state total energy takes its minimum at the true density. According to the Kohn-Sham theory [24] there exists a non-interacting system, where the electrons move independently in a common, local (Kohn-Sham) potential.

Introduce a phase-space distribution function $f(\mathbf{r}, \mathbf{p})$ with the properties:

$$
\begin{aligned}
& \int d \mathbf{p} f(\mathbf{r}, \mathbf{p})=n(\mathbf{r}), \\
& \int d \mathbf{r} n(\mathbf{r})=N,
\end{aligned}
$$

and

$$
\int d \mathbf{p} \frac{p^{2}}{2 m} f(\mathbf{r}, \mathbf{p})=t_{s}(\mathbf{r})
$$

$m$ is the mass. The integral of the non-interacting kinetic energy density $t_{s}(\mathbf{r})$ gives the non-interacting kinetic energy $E_{k i n}$

$$
E_{k i n}=\int d \mathbf{r} t_{s}(\mathbf{r})
$$


Several distribution functions satisfy the marginal conditions (12-14). The general forms of distribution functions satisfying somewhat different marginal conditions were studied by Cohen [25]. Ghosh, Berkowitz and Parr selected a distribution function by maximizing the entropy

$$
\begin{gathered}
S=\int d \mathbf{r} s(\mathbf{r}) \\
s(\mathbf{r})=-k \int d \mathbf{p} f(\ln f-1)
\end{gathered}
$$

subject to the constraints of correct density ( Eq. (12)) and correct non-interacting kinetic energy ( Eq. (14)). $k$ is the Boltzmann constant. They obtained a local Maxwell-Boltzmann distribution function

$$
f(\mathbf{r}, \mathbf{p})=e^{-\alpha(\mathbf{r})} e^{-\beta(\mathbf{r}) p^{2} / 2 m},
$$

where $\alpha(\mathbf{r})$ and $\beta(\mathbf{r})$ are $\mathbf{r}$-dependent Lagrange multipliers. The local temperature $T(\mathbf{r})$ is defined in terms of the non-interacting kinetic energy density

$$
t_{s}(\mathbf{r})=\frac{3}{2} n(\mathbf{r}) k T(\mathbf{r})
$$

i.e. by the ideal gas expression. Eqs. (14), (18) and (19) lead to

$$
\beta(\mathbf{r})=\frac{1}{k T(\mathbf{r})}
$$

and

$$
f(\mathbf{r}, \mathbf{p})=[2 \pi m k T(\mathbf{r})]^{-3 / 2} n(\mathbf{r}) e^{-p^{2} / 2 m k T(\mathbf{r})} .
$$

It can be rewritten as

$$
f(\mathbf{r}, \mathbf{p})=e^{\mu / 2 k T(\mathbf{r})} e^{-\left(p^{2} / 2 m+v_{e f f}\right) / k T(\mathbf{r})},
$$

where

$$
v_{e f f}=\mu-\frac{\ln \lambda^{3} n}{\beta}
$$

and

$$
\lambda=\frac{1}{2 \pi m k T}
$$

Note that this effective potential $v_{\text {eff }}$ is not the same but related to the Kohn-Sham potential [4]. 


\section{FISHER INFORMATION AND QUANTUM SYSTEMS}

Now we turn to the quantum mechanical generalization of the Pennini-Plastino theory [3]. First define the phase-space distribution function $\varrho(\mathbf{r}, \mathbf{p})$ as

$$
\varrho(\mathbf{r}, \mathbf{p})=\frac{f(\mathbf{r}, \mathbf{p})}{N}=\frac{e^{-\beta h_{e f f}}}{N}
$$

where

$$
h_{e f f}=\frac{p^{2}}{2 m}+v_{e f f}-\mu .
$$

$N$ appears in the definition of $\varrho(\mathbf{r}, \mathbf{p})$ because the density integrates to the number of electrons $N$ ( Eq. (13)) in the density functional theory, while $\varrho(\mathbf{r}, \mathbf{p})$ is normalized to 1.

Differentiation of $\varrho(\mathbf{r}, \mathbf{p})$ leads to the quantum mechanical counterparts of the classical canonical equations (7) and (8):

$$
\frac{\partial \ln \varrho}{\partial p_{i}}=-\beta \frac{\partial h_{e f f}}{\partial p_{i}}
$$

and

$$
\frac{\partial \ln \varrho}{\partial x_{i}}=-\frac{\partial\left(\beta h_{e f f}\right)}{\partial x_{i}}=-\beta\left[\frac{\partial h_{e f f}}{\partial x_{i}}+h_{e f f} \frac{\partial \ln \beta}{\partial x_{i}}\right],
$$

$i=1,2,3$. Equations (27) have exactly the same form as the classical ones (Eq. (7)). Eqs. (28) are different from the classical ones because of the position dependence of the local temperature.

The phase space Fisher information takes the form

$$
I=\int d \mathbf{r} d \mathbf{p} \varrho\left[a\left(\nabla_{\mathbf{p}} \ln \varrho\right)^{2}+b\left(\nabla_{\mathbf{q}} \ln \varrho\right)^{2}\right] .
$$

The coefficients $a$ and $b$ are included due to dimensional reasons. Taking into accout of Eqs. (27) and (28) we are led to

$$
I=\beta^{2} \int d \mathbf{r} d \mathbf{p} \varrho\left[a\left(\nabla_{\mathbf{p}} h_{e f f}\right)^{2}+b\left(\nabla_{\mathbf{r}} h_{e f f}+h_{e f f} \nabla_{\mathbf{r}}(\ln \beta)\right)^{2}\right] .
$$

Comparing Eq. (30) with the classical one (Eq. (10)) we can see that there is an extra term (the last one in Eq. (30)) due to the position dependence of $\beta$. In certain special cases it may happen that the $\beta$ is constant (see the following section). Then the phase space Fisher information has the same form as the classical one:

$$
I=\beta^{2} \int d \mathbf{r} d \mathbf{p} \varrho\left[a\left(\nabla_{\mathbf{p}} h_{e f f}\right)^{2}+b\left(\nabla_{\mathbf{r}} h_{e f f}\right)^{2}\right] .
$$

In the following section two examples are presented. 


\section{EXAMPLES AND DISCUSSION}

Consider first the example of the linear harmonic oscillator. The potential is $V=\frac{1}{2} m \omega^{2} x^{2}$, where $m$ is the mass and $\omega$ is the frequency. The ground-state density is given by

$$
n(x)=\left(\frac{m \omega}{\pi \hbar}\right)^{1 / 2} e^{-m \omega x^{2} / \hbar} .
$$

The kinetic energy density

$$
t(x)=\frac{\hbar^{2}}{2 m}\left(\frac{d \psi}{d x}\right)^{2}+c \nabla^{2} n
$$

is not uniquely defined. ( $\psi$ is the wave function.) Any value of $c$ gives the proper kinetic energy as the Laplacian of the density $n$ integrates to 0 . After elementary calculation we are led to

$$
t(x)=\hbar \omega n\left[\frac{m \omega}{2 \hbar} x^{2}(1+4 \tilde{c})-\tilde{c}\right]
$$

where $\tilde{c}=2 m c / \hbar^{2}$. With the choice of $\tilde{c}=-1 / 4$ we obtain

$$
t(x)=\frac{1}{4} \hbar \omega n(x) .
$$

Instead of Eq. (19) we have now

$$
t(x)=\frac{1}{2} k \operatorname{Tn}(x)=\frac{n(x)}{2 \beta}
$$

as the degrees of freedom is one in the present example. Therefore we have a constant inverse temperature:

$$
\beta=\frac{2}{\hbar \omega}
$$

or $k T=\hbar \omega / 2$, that is, the temperature corresponds to the ground-state energy of the oscillator. From Eq. (31) the phase-space Fisher information can be given by

$$
I=\beta\left(a \frac{1}{m}+b m \omega^{2}\right) .
$$

The choice $a=m / \beta_{0}$ and $b=1 /\left(m \omega^{2} \beta_{0}\right)$ leads to

$$
I=2 \frac{\beta}{\beta_{0}}
$$


in agreement to the classical result [3], where $\beta_{0}$ is an arbitrary, fixed reference inverseenergy. As the ground-state energy is $E=\hbar \omega / 2$ the phase-space Fisher information can also be written as

$$
I=2 \frac{\beta^{2}}{\beta_{0}} E
$$

in accordance with the classical expression [3].

As a second example consider the Hydrogen atom in its ground state. The density is given by

$$
n(r)=\frac{1}{\pi}\left(\frac{Z}{a_{0}}\right)^{3} e^{-2 Z r / a_{0}},
$$

where

$$
a_{0}=\frac{\hbar^{2}}{m e^{2}}
$$

and $Z, r, m$ and $e$ are the atomic number, the radial distance, the electron mass and the magnitude of the electronic charge, respectively. The kinetic energy density is not uniquely defined. We select the gradient form

$$
t(r)=\frac{\hbar^{2}}{2 m}(\nabla \psi)^{2}
$$

where $\psi$ is the ground-state wave function. Adding a term proportional to the Laplacian of the density would result a kinetic energy density with the same kinetic energy but different local temperature. Expression (43) leads to

$$
t(r)=\frac{\hbar^{2} Z^{2}}{2 m a_{0}^{2}} n(r),
$$

therefore the local temperature is constant:

$$
k T=\frac{1}{\beta}=\frac{Z^{2} m e^{4}}{3 \hbar^{2}} .
$$

Using Eq. (31) the phase-space Fisher information has the form

$$
I=\beta\left(3 a \frac{1}{m}+b \frac{4}{3} \frac{Z^{4} m^{3} e^{8}}{\hbar^{6}}\right) .
$$

Selecting $a=m / \beta_{0}$ and $b=\hbar^{6} /\left(Z^{4} m^{3} e^{8} \beta_{0}\right)$ we are led to

$$
I=\frac{13}{3} \frac{\beta}{\beta_{0}}
$$


We have to emphasize that the "thermodynamical" transcription is done for the quantum mechanical ground state. Therefore the notation 'temperature' is strictly speaking an analogy. As it was already mentioned the local kinetic energy is not uniquely defined. We can take advantage of this freedom. We can always select a form for the local kinetic energy which is everywhere positive resulting in a positive temperature. Though generally the local temperature depends on the position, in special cases it might be constant. The examples presented above are such cases. In these examples the phase-space Fisher information has an analitical form. In other cases or other expressions of the local kinetic energy the phasespace Fisher information can only be numerically determined that can be the subject of further investigation.

In the classical case the temperature is a parameter independent of the system considered. In the quantum mechanical theory presented here, however, the 'temperature' is an inherent property of the system as it is determined by the local kinetic energy. Therefore the 'temperature' depends on characteristic parameters of the system, e.g on the frequency of the harmonic oscillator and on the atomic number of the Hydrogen atom.

In summary, a quantum mechanical generalization of the Pennini - Plastino theory is presented making use of the thermodynamical transcription of the density functional theory. Comparing to the classical case, the phase-space Fisher information contains an extra term due to the position dependence of the temperature. However, if the temperature is constant, the expression derived bears resemblance to the classical one. This complete analogy to the classical case is exposed for the linear harmonic oscillator.

\section{Acknowledgments}

The grant OTKA No. K 100590 is also gratefully acknowledged.

[1] R. A. Fisher, Proc. Cambridge Philos. Soc. 22 (1925) 700.

[2] R. Frieden, Physics from Fisher Information. A unification; Cambridge: U. P., 1998.

[3] F. Pennini and A. Plastino, Phys. Lett. A 349 (2006) 15.

[4] S.K. Ghosh, M. Berkowitz and R.G. Parr, Proc. Natl. Acad. Sci. USA 81 (1984) 8028.

[5] S. K. Ghosh and R. G. Parr, Phys. Rev. A 34 (1986) 785. 
[6] S.K. Ghosh and M. Berkowitz, J. Chem. Phys. 83 (1985) 2979.

[7] S. R. Gadre and R. D. Bendale, Int. J. Quant. Chem. 28, 311 (1985).

[8] S. R. Gadre, S. B. Sears, S. J. Chakravorty and R. D. Bendale, Phys. Rev. A 32, 2602(1985).

[9] S. R. Gadre, Phys. Rev. A 30, 620(1984).

[10] S. R. Gadre, S.A. Kulkani and I. H. Shrivastava, Chem. Phys. Lett.166, 445 (1990).

[11] S. R. Gadre, R. D. Bendale, S. P. Gejii, Chem. Phys. Lett.117, 138 (1985).

[12] Á. Nagy and R. G. Parr, Proc. of Ind. Acad. Sci. (Chem. Sci) 106 (1994) 217.

[13] Á. Nagy and R. G. Parr, J. Mol. Struct. (Theochem) 501 (2000) 101.

[14] Á. Nagy and R. G. Parr, Int. J. Quantum Chem. 58 (1996) 323.

[15] Á. Nagy, Proc. of Ind. Acad. Sci. (Chem. Sci) 106 (1994) 251.

[16] Á. Nagy, in Reviews of Modern Quantum Chemistry, ed. K. D. Sen (World Scientific, 2002) Vol.I, p.413.

[17] Á. Nagy, J. Mol. Struct Theochem 943 (2010) 48-52.

[18] F. Pennini and A. Plastino, Phys. Rev. E 69 (2004) 057101.

[19] R. Frieden, A. Plastino, A. R. Plastino and B. H. Soffer, Phys. Rev. E 60 (1999) 48.

[20] R. Frieden, A. Plastino, A. R. Plastino and B. H. Soffer, 66 (2002) 046128.

[21] R. Frieden, A. Plastino, A. R. Plastino and B. H. Soffer, Phys. Lett. A 304 (2002) 73.

[22] F. Olivares, F. Pennini and A. Plastino, Phys. A 389 (2010) 2218.

[23] P. Hohenberg and W. Kohn, Phys. Rev. 136 (1964) B864.

[24] W. Kohn and L. J. Sham, Phys. Rev. 140(1965) A1133.

[25] L. Cohen, J. Chem. Phys. 80 (1984) 4277. 